\title{
RECOLECCIÓN Y REUTILIZACIÓN DE LAS AGUAS DE LLUVIA EN EDIFICIOS COMO BENEFICIO PARA LAS CIUDADES
}

Eje 2: Tecnología para la construcción sustentable

Demartini Juan Ignacio'

Bertoni Griselda Alicia ${ }^{2}$

Piga Lisandro ${ }^{3}$

\author{
${ }^{1}$ Pasante en la materia "Forma, Materiales y Proyecto"; FADU UNL; Santa Fe, Argentina, \\ juanignaciodemartini@gmail.com \\ ${ }^{2}$ Responsable a cargo de la materia y Directora del Proyecto; FADU UNL; Santa Fe, Argentina, \\ www.fadu.unl.edu.ar; arqgbertoni@gmail.com \\ ${ }^{3}$ Pasante en la materia "Forma, Materiales y Proyecto"; FADU UNL; Santa Fe, Argentina, \\ lisandropiga@gmail.com
}

\section{RESUMEN}

La investigación surge de la problemática que afrontan las ciudades debido a las intensas precipitaciones, donde el cambio climático, el continuo crecimiento, obsoletos sistemas de infraestructura de desagües llevan a colapsar los sistemas de evacuación de aguas de lluvia de las ciudades durante las precipitaciones intensas

El presente trabajo busca señalar que el conflicto del agua en Santa Fe es un problema heterogéneo que no puede resolverse de forma aislada, sino que debe abordarse a través de un método más complejo, inter-disciplinar y variable. Aportar a la resolución del problema de anegamiento que produce el agua de lluvia, por medio de la recolección, reutilización y/o evacuación a través de los retardadores u otros sistemas al interior del predio, contribuirá a la solución del problema existente. De esta forma, disminuirá el caudal excedente en los medios de escurrimiento, reduciendo los impactos negativos en la ciudad y otorgando beneficios de tipo sustentable a este recurso natural.

La superficie de las ciudades que anteriormente eran permeables, hoy ya no lo son, motivo por el cual las capacidades naturales del territorio se ven alteradas. Tomar los problemas por separado simplifica el intento de solución, ya que al entender esto solo como un problema de inundación urbana, se ignoran la contaminación del agua, la calidad del aire, el efecto de isla de calor urbano, etc.

Ante esta situación se hace necesaria una mirada compleja, que establezca relaciones, admita la incertidumbre y mantenga una continua evolución; porque si bien las infraestructuras deben seguir el crecimiento de la ciudad, ambas se encuentran en continuo proceso de cambio. 
Entendemos que los retardadores no convencionales son fáciles de combinar con los convencionales, permitiendo potenciar sus resultados no solo a nivel de evacuación pluvial urbana (posibilidad de absorber- retener el 100\%), sino también aportando a la consideración ecológica (Ej: reducción en la emisión de calor de las cubiertas naturadas, con devolución al ambiente del agua por el proceso de evapotranspiración) y también las de tipo sustentable (Ej: reciclado de agua con la captación y almacenado de un tanque de reserva).

La validación se realiza a partir de la intervención en un edificio existente, esto permite ensayar la factibilidad de aplicación de los distintos sistemas de reguladores pluviales analizados.

\section{PALABRAS CLAVES: RETARDADOR - PLUVIAL - REUTILIZACIÓN - AGUA}

\section{INTRODUCCIÓN}

La investigación surge de la problemática que afrontan las ciudades debido a las intensas precipitaciones, donde el cambio climático, el continuo crecimiento, obsoletos sistemas de infraestructura de desagües llevan a colapsar los sistemas de evacuación de aguas de lluvia de las ciudades durante las precipitaciones intensas

La mayor cantidad de metros cuadrados construidos provocan el aumento de superficies impermeables, lo que acarrea una reducción de la absorción natural del suelo y la posterior acumulación indeseada de considerables cantidades de agua en sectores de menor cota; dando lugar a inundaciones espontáneas en la ciudad.

"La urbanización provoca efectos y cambios importantes en el ciclo hidrológico, aumentando los volúmenes de escurrimiento, las descargas pico y reduciendo los tiempos de distribución del flujo". (Instituto Nacional del Agua [INA], 2013)

"En general las grandes ciudades del país se ven afectadas por un acelerado y desordenado crecimiento de la urbanización, aumentando los efectos mencionados y dejando a las redes existentes de desagüe pluvial sin capacidad para conducir los caudales generados y en consecuencia las inundaciones son cada vez más frecuentes." (INA, 2013)

El proceso de urbanización actual y el desarrollo de los grandes centros urbanos, en general se produce de una manera acelerada, descapitalizada y poco planificada; dando lugar a transformaciones económicas que agrandan la brecha entre los sectores sociales. Esto se ve reflejado en cuestiones tales como el hacinamiento, degradación ambiental, déficit habitacional cuantitativo y cualitativo, insuficiencias en materia de infraestructura, entre otras.

"La urbanización es creciente en el mundo y particularmente en Sur América. Esta tendencia, que también se registra en Argentina, genera continuas presiones para la ocupación del espacio urbano. La rápida expansión de los sectores más carenciados torna difícil el control de todos los aspectos asociados al crecimiento." (Silvio Ambrosino et al. 2004, pag. 13)

El Banco Mundial hace referencia al agua como amenaza producida por inundaciones y las clasifica en cuatro tipos básicos: 1. En los valles de los grandes ríos (o inundaciones ribereñas). 2. En el piedemonte andino (por rápido deshielo de nieves y/o lluvias concentradas). 3. Inundaciones relámpago ocurridas en ciudades y zonas rurales (generadas a partir de fuertes tormentas). 4. 
Inundaciones en áreas de llanuras, generadas por fuertes tormentas y originadas a partir del inadecuado drenaje y manejo del suelo rural, incluyendo en ello el tratamiento dado a la red vial de caminos secundarios rurales.

En Argentina, además de las inundaciones ribereñas (tipo 1), provocadas por el desarrollo urbano tradicional sobre valles aluviales (de inundación); se observan las provocadas por tormentas (tipo 3 y 4): generadas a partir de fuertes alteraciones del ambiente rural con fines agrícolas, como también por la falta de desarrollo en infraestructura vial principal y secundaria. Este tipo de inundaciones afectan a centros urbanos de mediano y pequeño porte y son típicas de la región agrícola central del país.

Las nuevas técnicas de control de inundación por lluvia, se basan en el principio de la desaceleración del escurrimiento en cuencas urbanizadas mediante sistemas de almacenamiento o infiltración, sistemas de compuertas para retener en las redes de desagüe a tiempo real, sistemas de almacenamiento en grandes túneles con bombeo posterior a la tormenta, o bien más ecológico, como lagos de retención integrados a las ciudades.

No obstante, aun contando con estas técnicas, en cuencas altamente urbanizadas, las soluciones propuestas son cada vez más complejas, debido a la falta de espacios verdes disponibles, si se opta por soluciones con almacenamiento. $Y$ en los casos de soluciones clásicas por conducción a superficie libre, surgen conflictos por la gran cantidad de interferencias de infraestructura de servicios públicos y de los impactos que producen estas obras al medio ambiente. Como por ejemplo el Proyecto "Plaza del agua" Rotterdam, o bien el Proyecto G-Cans Kasukabe, prefectura de Saitama, Japón.

Respecto de las precipitaciones, se deberá tener en cuenta que pueden tener diferentes destinos producto de su ciclo, una considerable parte es evaporizada y dirigida nuevamente a la atmósfera, gran parte se escurre por las superficies terrestres (escorrentía), generalmente por los cordones cunetas o zanjas (agudizada por el alto grado de impermeabilidad), y por último, la restante, es la que se infiltra en el terreno, pudiendo volver a la atmósfera por evapotranspiración, o bien alcanzar las napas freáticas.

Es decir que, no todos los factores que influyen en una inundación son producto de una mala planificación, el cambio climático, entendido como una variación significativa y persistente del clima durante un período largo de tiempo producido por causas naturales y humanas, también impacta en los regímenes hidrológicos.

Por otro lado, si bien se puede encontrar agua libremente en la naturaleza, el suministro, especialmente en grandes ciudades, se ha convertido en un reto para las empresas prestadoras del servicio; el consumo de energía y el costo que genera la extracción, potabilización y transporte son considerables para justificar una economía de uso. Aunque tengamos abundante agua, cada vez es más costoso potabilizarla para el uso doméstico debido al incremento de contaminación determinado por la directa proporción con el aumento de la población.

Debemos considerar que la cantidad media óptima de agua para consumo doméstico humano (beber, cocinar, higiene personal y limpieza del hogar) es de 100 litros por persona / día, pese a ello, en algunos países el consumo alcanza los 300, triplicando el mínimo considerado para cubrir las necesidades básicas. Es el principal e imprescindible componente del cuerpo humano, cumple un rol esencial en la alimentación, higiene, producción; debiendo ser incorporado al proyecto 
arquitectónico junto con el concepto de reciclado (almacenado y reutilización), aportando a la ecología, infraestructura, economía. Al mismo tiempo, que se puede convertirse en un factor estético primordial de diseño, cargarlo de significado y proporcionar de calidad sus espacios.

Por otra parte, la ciudad de Santa Fe está asentada entre dos ríos de llanura (Salado y Paraná), con sus valles de inundación, que en su rol natural, cumplen la función de atenuar las crecientes al permitir que el agua, en los picos de crecidas, se derrame sobre una zona más amplia que el propio cauce del río. Vale señalar que la ciudad para resguardarse de las crecidas, ha generado un anillo completo hoy (no así en el 2003) con cordones de defensa a cota segura, cuya problemática radica en la existencia de barrios que se encuentran asentados sobre dichos valles fuera de los cordones, y otra gran parte de los más afectados, sobre cotas inferiores a la segura.

De modo que esa vulnerabilidad es quien convierte la probabilidad de ocurrencia del fenómeno en una amenaza. Esa posibilidad de que ocurra un desastre, representa un riesgo, al conectar las circunstancias de amenaza y vulnerabilidad. Condición que deberían respetar los asentamientos y exigir los Gobiernos municipales y comunales. Asentarse en las zonas más bajas de la ciudad equivale a aumentar la vulnerabilidad ante la amenaza existente, haciendo más probable los riesgos de inundaciones.

Históricamente el principal problema en este orden, fue el desborde de los ríos, se padecieron inundaciones recurrentes, hasta que las defensas fueron repensadas y reconstruidas. Hoy el tema ha sido al menos controlado, reemplazándose por la acumulación de las aguas de lluvia, con su posterior necesidad de derivación. El propio recurso de las defensas conforma un recipiente contenedor, que ante las desmesuradas precipitaciones y el ineficiente desempeño del drenaje, provoca un conflicto de interés social, técnico y arquitectónico, que motivan su estudio.

Las defensas hidráulicas son acciones estructurales destinadas a conformar un sistema de protección de urbanizaciones. Obras de ingeniería diseñadas para atenuar el efecto de determinadas eventualidades naturales, son calculadas en base a datos estadísticos e históricos, para resistir acontecimientos de cierta probabilidad de ocurrencia. En Santa Fe coexisten: terraplenes perimetrales a los cursos de agua (que conforman espacios protegidos cerrados), estructuras de regulación de caudales, sistemas de reservorios (que actúen como retención provisional del agua), estaciones de bombeo, canales de desagüe, calles de pavimento articulado, alcantarillas, espacios verdes, zanjas a cielo abierto.

Dentro de las medidas no estructurales, cuenta con diferentes normativas (ROU) que establecen el tipo de actividad o destino para cada zona, exige tamaños mínimos de parcelas, infraestructura mínima requerida, el factor de ocupación total (FOT) y el Factor de Impermeabilización del Suelo (FIS). Además, desde fines del año 2012, la ordenanza 11.959, dispone la incorporación de sistemas de retardadores pluviales dentro del predio, los cuales permiten regular los excedentes para caudales máximos generados por futuras urbanizaciones y así mantengan los valores similares a los precedentes. 


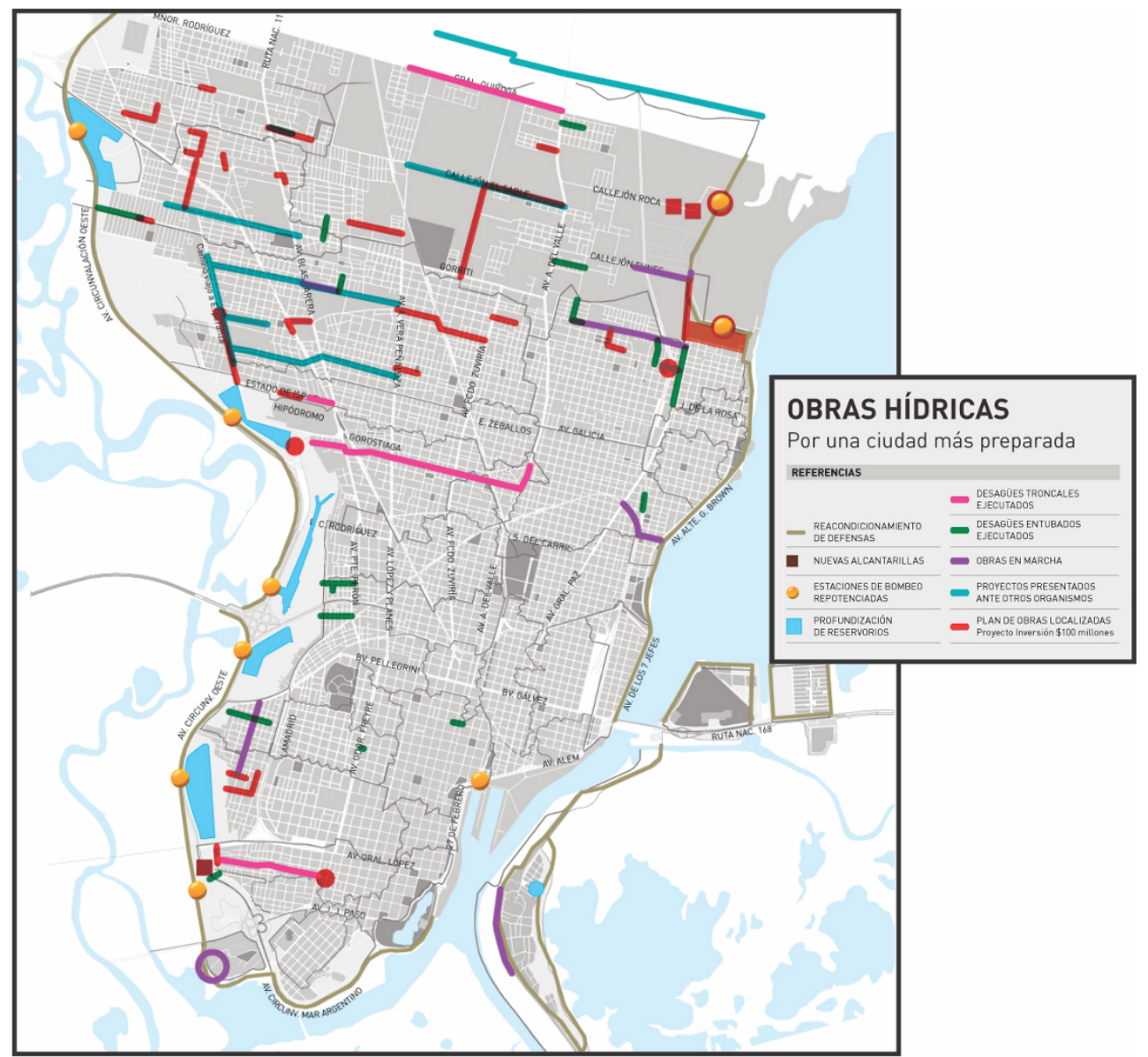

Fig. 1.1 (mapa de Santa Fe y los sistemas de defensa)

El presente trabajo busca señalar que el conflicto del agua en Santa Fe es un problema heterogéneo que no puede resolverse de forma aislada, sino que debe abordarse a través de un método más complejo, inter-disciplinar y variable. Aportar a la resolución del problema de anegamiento que produce el agua de lluvia, por medio de la recolección, reutilización y/o evacuación a través de los retardadores u otros sistemas al interior del predio, contribuirá a la solución del problema existente. De esta forma, disminuirá el caudal excedente en los medios de escurrimiento, reduciendo los impactos negativos en la ciudad y otorgando beneficios de tipo sustentable a este recurso natural.

Se encuentra que la regulación o aprovechamiento del agua puede conseguirse en diferentes escalas y niveles de intervención: a nivel de predio, con intervención del Propietario y del Municipio; a nivel de calles en el ingreso de agua a conductos, con intervención del Municipio; a nivel de cuenca urbana y rural en zonas apropiadas de almacenamiento, con intervención del Municipio y/o Provincia.

La regulación a nivel de predio es la que interesa específicamente, ya que corresponde a soluciones que el arquitecto debe ser capaz de adecuar aplicando los sistemas reguladores a la funcionalidad, diseño y economía de un edificio; sin dejar de lado la urbanización y su relación con el entorno. 


\section{DESARROLLO}

Se considera que un retardador emula el efecto que antes lograba la vegetación, actúa como un pulmón regulador haciendo que el agua se infiltre y no vaya directamente a la red. Esta superficie verde ya no existe o está disminuida, pero se puede recrear artificialmente buscando la manera de que el exceso de agua quede retenido en un determinado sector para después reciclarlo, retardarlo o devolverlo al medio ordenadamente, sin producir los efectos indeseables de concentración e inundación. Recolectar y reutilizar las aguas de lluvia permitiría reducir los caudales de evacuación de las mismas hacia los desagües públicos, permitiendo descongestionar los reservorios, reducir los volúmenes de metros cúbicos de líquidos en las bombas impulsoras y evitar el acumulamiento en las zonas más bajas de la ciudad. Por otro lado, se puede pensar en su acumulación y reserva, representando un beneficio para el medio ambiente.

Se presentan a continuación los distintos sistemas de retardadores/contenedores, sus variables, posibles combinaciones y el efecto de su aplicación Los podemos dividir en dos grandes áreas, absorbente y no absorbentes.

\section{1) RETARDADORES NO ABSORBENTES:}

a- Abiertos (Pisos / Cubiertas Azules / Bandejas modulares prefabricadas)

b- Cerrados (Cisternas)
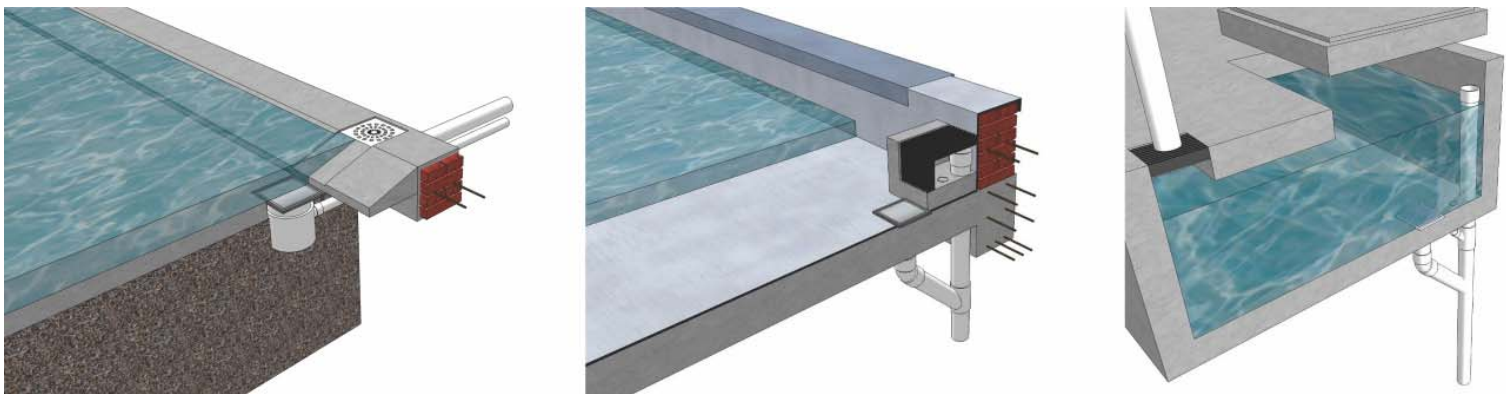

Fig. 2.1 (Esquemas constructivos de Izq. A Der.: pisos azules, cubiertas azules y cisternas)

1) a- Abiertos (pisos / cubiertas azules / bandejas): Se conocen como aquellos recipientes capaces de contener un determinado volumen de agua. Pueden ser de distintas profundidades dependiendo su uso, por ejemplo: espejos de agua, fuentes, piscinas, tanques de reservas contra incendios, bandeja contenedora. Estos admiten la posibilidad de combinarlos con sistemas de retardadores pluviales.

En estos, el agua de lluvia proveniente se va acumulando en estos recipientes o contenedores, hasta el momento que supera su máximo nivel, donde comienza a desbordar a través de elementos que posibilitan su desagüe.

Ventajas: Retienen hasta donde le permite su capacidad de almacenamiento, son de bajo mantenimiento, y pueden ser combinables con sistemas de reutilización o contra-incendios.

Desventajas: Espacio disponible (a excepción de las bandejas), estética desfavorable, obra moderada una vez alcanzada su vida útil. 
b- Cerrados (Cisternas): Es un sistema recipiente capaz de contener un determinado volumen de agua; al mismo tiempo que posibilita retener, reciclar y/o retardar su evacuación según el caso que corresponda. El agua de lluvia es recolectada por distintos medios, se va acumulando en un contenedor y al momento que es superado un determinado nivel, comienza a desbordar a través de elementos que posibilitan su desagüe. En el caso que su función sea la de regular los excedentes pluviales, el sistema contará con elementos de evacuación de secciones reducidas o de algún dispositivo que le permita ir eliminando menores cantidades de líquido a la red, con un retardo de tiempo.

Ventajas: Regular los caudales excedentes, bajo mantenimiento, de fácil aplicación, combinable con sistema de reutilización y sistema contra-incendios.

Desventajas: Espacio disponible, Estética desfavorable.

\section{2) RETARDADORES ABSORBENTES:}

a- Naturados (Pisos / Cubiertas Verdes / Cubiertas Marrones)

b- Artificiales i) Pavimentos Inter-trabados ii) Hormigones Permeables
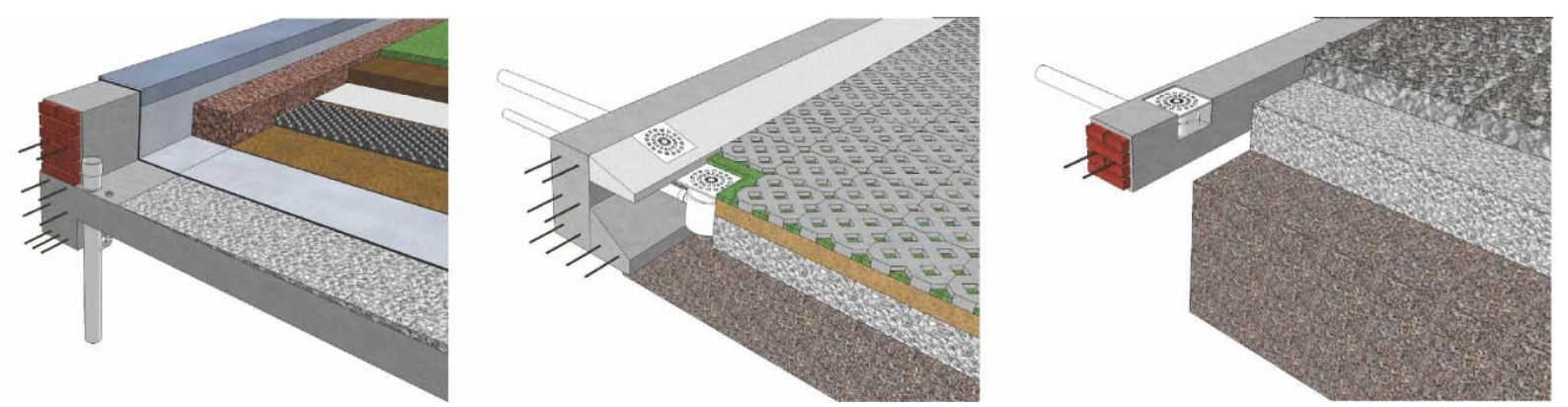

Fig. 2.2 (Esquemas constructivos de Izq. A Der.: cubiertas naturada, piso cribado y piso pav. poroso)

2) a- Naturados: Son aquellos sistemas que buscan satisfacer la necesidad de instalar vegetación sobre un techo. Incorporan una capa de sustrato, medio en el que se desarrolla la raíz del vegetal, retiene la humedad, intercambia gases y capta los nutrientes necesarios para su desarrollo.

Cierto porcentaje de la lluvia es retenido por el follaje de la vegetación. Parte del agua regresa a la atmósfera por evapotranspiración, mientras que otra es retenida por el sustrato de acuerdo con su composición. Una vez que el sustrato está saturado y las concavidades llenas, el agua sobrante puede fluir libremente hacia los desagües de la cubierta a través la pendiente y del elemento impermeabilizante.

Ventajas: Retención aprox. de 115Lts/m2 las intensivas y 30Lts/m2 las extensivas; mejoran la aislación acústica y térmica, emiten menor calor al ambiente, filtran la polución del agua y aire

Desventajas: Cubiertas pesadas $250 \mathrm{~kg} / \mathrm{m} 2$ (intensivas) y $120 \mathrm{~kg} / \mathrm{m} 2$ (extensivas) adicionales, mantenimiento (mayor en las intensivas), obra de consideración una vez alcanzada su vida útil.

b- i) Pavimentos inter-trabados: Se denomina pavimento a una capa constituida por uno o más materiales que se disponen sobre el terreno natural o nivelado, para aumentar su resistencia y servir para la circulación de personas o vehículos. 
Por el motivo que acontece, destacamos los entramados prefabricados de hormigón o "cribadosnaturados". Éstos al ser de "alma hueca" toleran el colado de agua. Su porcentaje de infiltración está representado por la diferencia de llenos y vacíos de la pieza. En cambio, el de absorción se determina según la capacidad de retención del sustrato a utilizar.

Ventajas: Materiales de alta resistencia, reutilizables, de rápida colocación, reducen la escorrentía, variables en colores y diseños.

Desventajas: Deben estar asociados con otros sistemas o sobre suelo natural, peso considerable.

ii) Hormigón permeable: Este adquiere su nombre debido a su alta porosidad y el contenido de vacíos vinculados. Dichos vacíos se distribuyen uniformemente en el interior del material, producto de los aditivos y la no utilización de áridos. Su porcentaje de vacíos puede variar entre el 15 y el $35 \%$; lo que representa el caudal de filtrado.

Ventajas: Materiales de alta resistencia, diferentes porcentajes de permeabilidad, reducen la escorrentía.

Desventajas: Deben estar asociados con otros sistemas o sobre suelo natural, peso considerable.

Para conocer su complejidad técnica se analizaron tres casos de tratamiento del excedente de agua de lluvia. Estos presentan características similares en cuanto al clima y geografía de la Ciudad de Santa Fe, pero se implantan en contextos Urbanos totalmente distintos. A su vez, el tratamiento del agua se manifiesta en diferentes escalas de sofisticación, lo que permite pensar en alternativas más creativas y complejas.

1) Ampliación del Instituto Universitario Nacional del Arte (IUNA), en Capital Federal: Esta ubicado en barrio Palermo, es la ampliación del antiguo edificio que se proyectó bajo la condición de un sistema de recolección, almacenado y reutilización del agua de lluvia.

La recolección de agua se realiza en los techos y su acumulación en un tanque ubicado en el subsuelo (cuyo volumen total es de 42.000 Its). Este tanque recibe, además de los 600 litros diarios de agua de lluvia, unos 1.800 litros diarios de aguas grises de los desagües de lavatorios; alimentando los depósitos de inodoros, cuya demanda estimada es de unos 2.300 litros diarios.

El agua de lluvia y las grises provenientes de los lavabos se vuelcan en el tanque de reserva subterráneo de reciclado, previamente habiendo pasado por interceptores y decantadores de sólidos. Desde allí, se alimenta a las mochilas de los inodoros, mingitorios y el sistema contra incendio. Esto se lleva a cabo gracias a una bomba de impulsión. En caso de desabastecerse dicho tanque, el agua es complementado por el de reserva de agua potable ubicado en la cubierta, quién además abastece los lavabos.

2) Estación de servicio YPF, en la Provincia de Buenos Aires diseñada por el estudio Hamptom Rivoira: Está situada en Nordelta, dentro del partido de Tigre. Se trata de un sector de lagos artificiales y naturales que dependen del agua pluvial para mantener su nivel. Los lagos se encuentran interconectados por conductos que autorregulan el nivel.

El edificio se desarrolla bajo una cubierta verde como articulador del programa arquitectónico.

Como aporte al ecosistema recupera el $100 \%$ de drenaje de la cubierta superior y el $50 \%$ de la playa de estacionamientos. La cual se almacena en un tanque de $11 \mathrm{mil}$ litros, para luego 
reutilizarse. La recuperación de agua de lluvia se produce al retener una parte para riego / lavado y la otra, con un desborde directo hacia el lago artificial para no afectar su volumen de agua. Lo recolectado se almacena en cisternas enterradas debajo del talud próximo a la sala de máquinas. Se retienen aguas grises de baños y vestuarios, además de la recuperación del agua proveniente de las rejillas del sector de autos. Mientras tanto, las aguas negras son evacuadas directamente al sistema de red primario y la captación por derrame combustibles a tratamiento correspondiente.

Se trata de un doble sistema de alimentación en baños y vestuarios (potable para duchas y lavatorios, y recicladas para grises como inodoros y mingitorios) En caso de insuficiencia se retroalimenta con agua de red.

3) Jardín de infantes Barranquitas, en la Ciudad Santa Fe: El proyecto, cuenta con un sistema de influencia directa sobre las inundaciones provocadas por excesos de precipitación.

Se trata de un retardador pluvial resuelto a través de un sistema adicional al desagüe pluvial convencional. Donde el agua de lluvia que cae en los techos se redirige por la misma fuerza gravitacional hacia los patios internos que posee el edificio. De allí, una determinada cantidad es absorbida por la tierra y el césped, y la que no lo alcanza es captada por rejillas de desborde; quienes se encuentran conectadas a una red de desagüe pluvial corriente.

Dichos conductos convergen en dos cámaras de inspección que se sitúan fuera del edificio, encargados de filtrar los posibles residuos que arrastre el agua y de retardar el flujo del mismo. Para aumentar la capacidad de almacenaje de dichos depósitos, se le incorporó al sistema un gran tubo de PVC de 600mm de sección que conecta ambas. El proceso se completa cuando el fluido comienza a salir por tubos de menor sección (PVC 63mm); a través de la presión misma, la gravedad e inclinación dispuesta hacia las cunetas correspondientes.

Como reflexión a los ejemplos analizados, podemos decir que los reservorios no convencionales son fáciles de combinar con los convencionales y permiten potenciar sus resultados no solo a nivel de evacuación pluvial urbana (dependiendo de la cantidad de lluvia, el sustrato y/o capacidad del reservorio, pueden absorber- retener el $100 \%$ de la misma), sino también aportar beneficios de tipo ecológicos (Ej: reducción en la emisión de calor de las cubiertas naturadas y devolución del agua al ambiente por proceso de evapotranspiración) y/o sustentables (Ej: reciclado de agua con la captación y almacenado de un tanque de reserva).

Finalmente se procedió a la elaboración de un proyecto para un edificio construido con el desafío de implementar la "evacuación cero". Es así que se busca un equilibrio de todas las variables de diseño, buscando aplicar las investigaciones precedentes e indagando beneficios sociales, ambientales, económicos y estéticos.

El edificio ensayado se encuentra en la Ciudad de Santa Fe, cuenta con un terreno de $256 \mathrm{~m} 2$ y la superficie construida es de $1521 \mathrm{~m} 2$, en diez pisos, combinando una oficina privada y nueve departamentos de uno, dos y tres dormitorios. La planta baja está destinada a hall y cocheras.

Para realizar comparaciones mensurables, se tuvo en cuenta la Ordenanza 11.959, que establece un retardo del $50 \%$ agua caída, con una intensidad de $60 \mathrm{~mm} / \mathrm{h}$, sobre la superficie impermeable (185m2, en nuestro caso). Es decir, si en una hora se mantiene dicha intensidad constante, sobre la superficie impermeable descripta, se recolectarán 11100 litros de agua. 

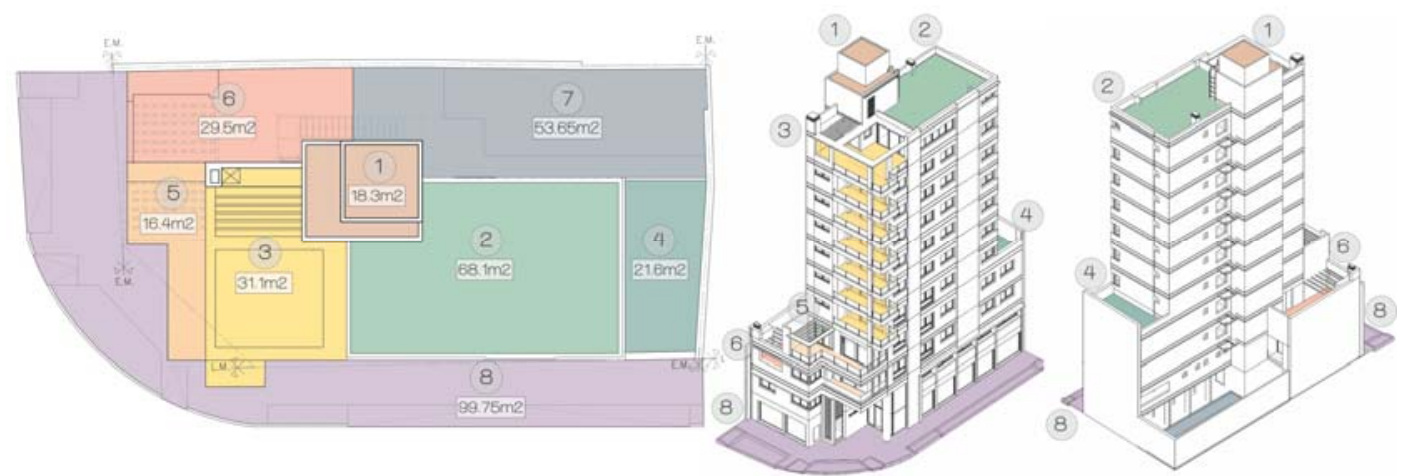

Fig. 2.3 (Esquemas de planta de techo y perspectivas sobre distintas áreas con posibilidad de intervención)

En la propuesta se detectan ocho sectores capaces de ser aprovechados para la captación de agua de lluvia, permitiendo realizar tres proyectos, con tres sistemas de reservorios distintos. Los aplicados fueron: el sistema de retardador convencional, el reservorio para reciclado con retardador y el sistema naturado con retardador.

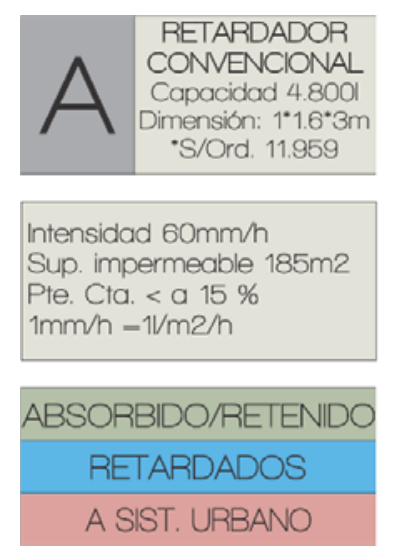

Fig. 2.4 (Cuadro comparativo sob
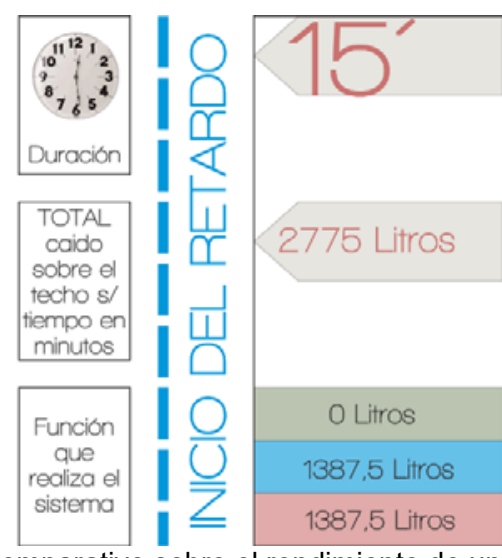
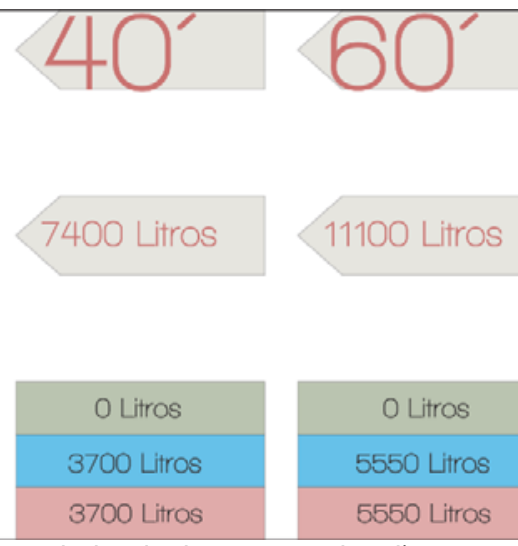

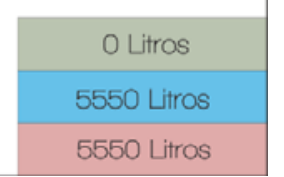

A- Se aprecia que sin importar el periodo de tiempo que llueva, el retardo comienza desde el inicio de la precipitación al $50 \%$, mientras que la otra mitad se evacúa a sistema urbano. Al cabo de los 60 minutos se evacuaron al sistema urbano 5.550 litros, retardando otros 5.550, hasta llegar a los 11.100 litros. Vale indicar que, superada esa intensidad, el retardo pierde la función por desborde y la totalidad del agua caída es vertida al sistema de red pluvial.

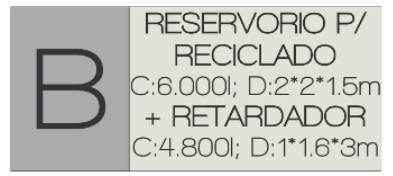

Intensidad $60 \mathrm{~mm} / \mathrm{h}$

Sup. impermeable $185 \mathrm{~m} 2$

Pte. Cta. < a $15 \%$

$1 \mathrm{~mm} / \mathrm{h}=1 / 1 / \mathrm{m} 2 / \mathrm{h}$

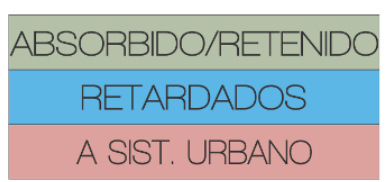

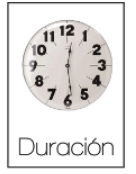
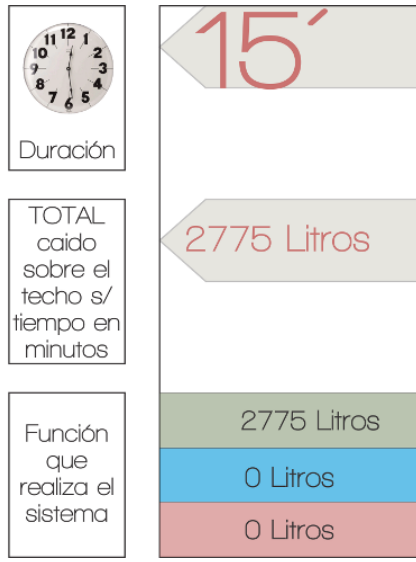
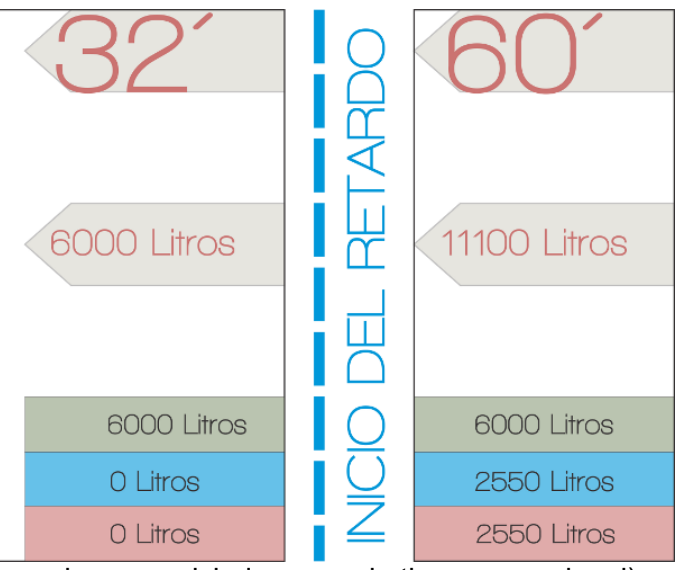

Fig. 2.5 (Cuadro comparativo sobre el rendimiento de un reservorio con reciclado + uno de tipo convencional) 
B- Durante los primeros 32 minutos el agua se acumula en el tanque de reserva adicional, para luego de dicho lapso comenzar el proceso de retardo del $50 \%$ de tipo convencional. En este caso 6000 litros (1.200 más de lo que pide retardar la ordenanza) son retenidos para el reciclado, 2550 litros van siendo evacuados al sistema urbano, mientras otros 2550 se van retardando, hasta llegar a los 5.100 litros. Es decir, solo con el reservorio se está cubriendo una lluvia de $60 \mathrm{~mm}$, sin importar la duración de la precipitación; además de no verter ningún excedente al sistema de red pluvial.
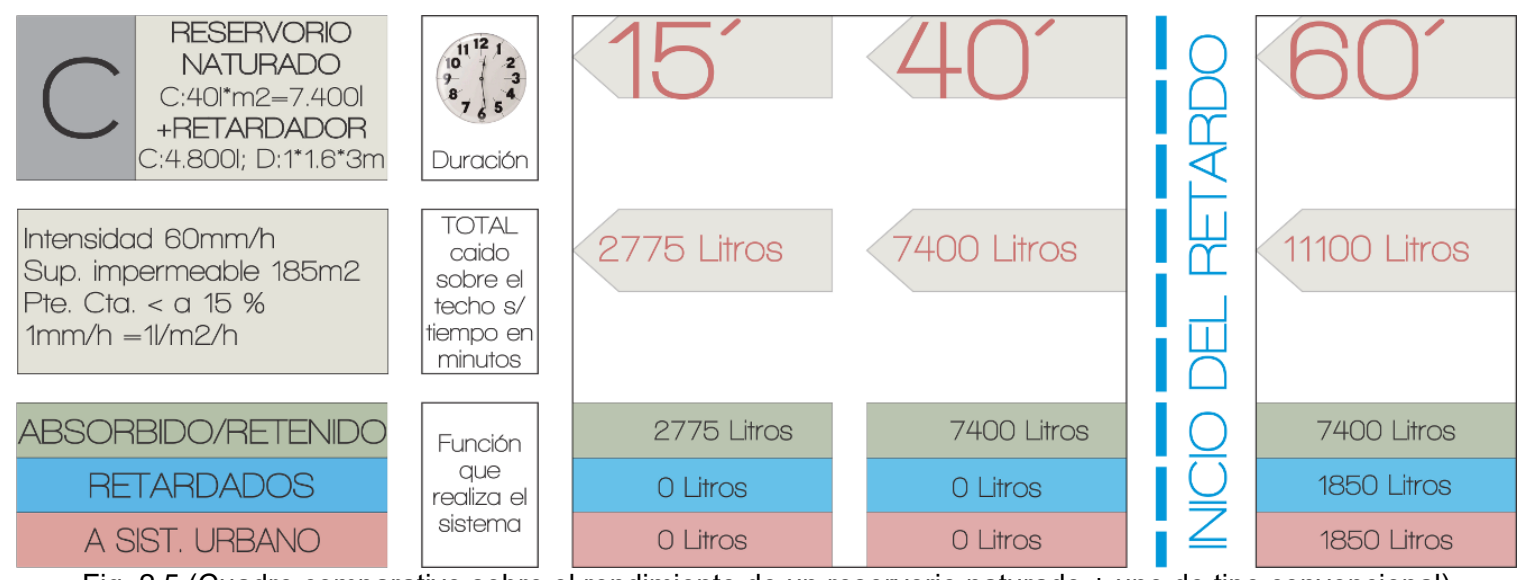

Fig. 2.5 (Cuadro comparativo sobre el rendimiento de un reservorio naturado + uno de tipo convencional)

C- Durante los primeros 40 minutos, hasta saturarse el sustrato, se absorbe el $100 \%$ de la lluvia. En este caso 7.400 litros de los 11.100 caídos, son absorbidos (2.600 más de lo que pide retardar la ordenanza), mientras que 1.850 se van evacuando, 1.850 se van retardando, hasta llegar a los 3.700 litros. Solo con lo absorbido se estaría cubriendo una lluvia de $60 \mathrm{~mm}$, sin importar la duración de la precipitación; además de no verter ningún excedente al sistema de red pluvial.

Como hemos visto, los reservorios no convencionales son fáciles de combinar con los convencionales y permiten potenciar sus resultados no solo a nivel de evacuación pluvial urbana (dependiendo de la cantidad de lluvia, el sustrato y/o capacidad del reservorio, pueden absorberretener el $100 \%$ de la misma), sino también aportar beneficios ecológicos (reducción en la emisión de calor de las cubiertas naturadas y devolución del agua al ambiente por evapotranspiración) y/o sustentables (reciclado de agua con la captación y almacenado de un tanque de reserva).

\section{CONCLUSIONES}

Es indudable que las capacidades naturales del territorio han sido alteradas, modificando su comportamiento frente a los regímenes de lluvia actuales. Frente a esto la solución es simplificada al buscar resolver solo un problema de inundación urbana, sin importar la contaminación del agua, la calidad del aire, o el efecto de isla de calor urbano, etc. Ante esto es necesario abordar el problema interdisciplinariamente, tener una mirada compleja de la situación, que establezca relaciones, admita la incertidumbre y mantenga una continua evolución

Vemos como actualmente se intensifica una crisis de sustentabilidad, no referida únicamente a la de los recursos naturales y por el hecho de no querer reutilizar agua de lluvia en una ciudad donde abundan los ríos. Sino a la sustentabilidad política, social y productiva, con un deterioro creciente de la calidad de vida (Fernandez, 2001). 
Esto se ve reflejado en normativas como la ordenanza $\mathrm{N}^{\mathrm{a}} 11.959$ (retardadores pluviales), que busca realizar aportes a nivel del predio, con la dificultad del control a posteriori de la obra civil.

Si bien el municipio exige su proyecto para la tramitación del permiso de obra e incluso no otorgar el Certificado Final si el sistema no se encuentra en regla, una vez que se entregada la misma, debería realizar controles para garantizar su correcto funcionamiento.

Es por estas razones que creemos oportuno aportar soluciones que complementen y optimicen los sistemas de prevención mencionados, de modo de generar conciencia en el ciudadano, incentivándolo a tomar iniciativas que ayuden a un plan general.

La incorporación de políticas y convenios económicos-financieros para dicho sector, que alienten la incorporación de tecnologías y recursos que aporten a la solución del problema, contribuirían a resultados observables en un lapso menor de tiempo.

\section{BIBLIOGRAFÍA}

ÁLVAREZ LEIVA, Carlos. Múltiples víctimas y catástrofes. Ed. ARAN. Madrid, España, 2008.

AMBrosino, S. BARBEITO, O. BERTONI, J.C. DANIELE, A. MAZA, J.A. PAOLI, A. SERRA, J.J.“Inundaciones urbanas en Argentina Córdoba, Argentina, 2004.

FATTORELLI, Sergio Y FERNÁNDEZ, Pedro C. Diseño hidrológico, Edición digital, 2011.

FERNANDEZ, ROBERTO, Derivas. Arquitectura en la cultura de la posmodernidad. De la modernidad imperfecta a la globalización salvaje. Argentina, 2001.

FLOR PÉREZ, José I. Hablemos del medio ambiente. PEARSON EDUCACIÓN, S.A. Madrid, España, 2006.

GOBIERNO DE LA CIUDAD DE SANTA FE, SECRETARÍA DE CULTURA Santa Fe, la ciudad y el río 2009.

HARDOY, Jorge E.; SATTERTHWAITE, David. La ciudad legal y la ciudad ilegal. Grupo Editor Latinoamericano, Colección Estudios Políticos y Sociales. Buenos Aires, Argentina, 1987

HOUGH, M. Naturaleza y ciudad. Gustavo Gili, S.A. Barcelona, 1998.

MORANT SÁNCHEZ, Carmen. Sensibilización medioambiental. Situación actual, problemática y búsqueda de soluciones. Ideas propias. Vigo, España, 2008.

NACIONES UNIDAS (CELADE - CEPAL). América Latina: Proyecciones de población urbano rural 1970-2025. Boletín Demográfico Nº3. Santiago, Chile. 1999. pag. 256.

O.P.S, O.M.S; Emergencias y desastres en sistemas de agua potable y saneamiento: guía para una respuesta eficaz. Washington, D.C. OPS, 2001.

ONU WATER PUBLICATIONS, Combatir la escasez de agua. El desafío del Siglo XXI. ONUAgua, FAO. 2000 
PAOLI, Carlos; 2000; Crecidas e inundaciones: un problema de gestión. Simposio las inundaciones de la República Argentina. Academia nacional de geografía - UNNE. Resistencia, Chaco, 2003.

PELLETIER, J. Y DELFANTE, CH. Villes et urbanisme dans le monde, $4^{\circ}$ édicion, Ed. A. Colin, Paris. 2000.

PESCl, Rubén; PÉREZ, Jorge; PESCI, Lucía. Proyectar la Sustentabilidad. Editorial CEPA. Buenos Aires, Argentina, 2007.

PROGRAMA DE LAS NACIONES UNIDAS PARA EL DESARROLLO, Informe sobre Desarrollo Humano 2006: Más allá de la escasez: Poder, pobreza y crisis mundial del agua. PNUD, 2006

RADOVANOVIC, E. Y TARTARINI, J. Agua y Saneamiento en Buenos Aires 1580. 1930, Proyecto Patrimonio Histórico CONICET-Aguas Argentinas, Bs. As. 1999.

RUBÉN MARCELO MOLINA. Lluvia, río y ciudad. Revista Origen / 56. Santa Fe, Argentina. 2013

SECCHI, A.; GIACOSA, R.; BIANCHI, H. Y MAZZÓN, R. Nuevas tecnologías para contribuir a la solución de las inundaciones en grandes ciudades, $1^{\circ}$ Seminario de drenagem urbana do Mercosul, P.Alegre, Brasil. (2001).

WILCHES-CHAUX, Gustavo. Auge, caída y levantada de Felipe Pinillo, mecánico y soldador o yo voy a correr el riesgo. Guía de La Red para la gestión local del riesgo. Popayán, Colombia, 1998.

WOLANSKY, Silvia, CORZO, Héctor, VALSAGNA, Andrea, MORBIDONI, Norberto (2003) Las inundaciones en Santa $\mathrm{Fe}$, Tomos 1 y 2 de la serie de Manuales Terraplenes de defensa y medidas de mitigación. Centro de Publicaciones de la Universidad Nacional del Litoral: Santa Fe.

ZINZI, M.; AGNOLI, S. Cool and Green Roofs. Energy and Buildings (In Press). Editorial Elsevier Science S. A. 11 p. 201.

\section{Páginas web}

http://www.ina.gov.ar/

http://www.argos.co/Colombia

http://www.ecoconcreto.com.mx/proceso.html

http://www.zinco-cubiertas-ecologicas.es/ 